\title{
Galanin Protects Against Behavioral and Neurochemical Correlates of Opiate Reward
}

\author{
Jessica J Hawes ${ }^{1,5}$, Darlene H Brunzell ${ }^{1,5}$, Roopashree Narasimhaiah ${ }^{1,5}$, Ülo Langel ${ }^{2}$, David Wynick ${ }^{3,4}$ \\ and Marina R Picciotto*,1
}

'Department of Psychiatry, Yale University School of Medicine, New Haven, CT, USA; ${ }^{2}$ Department of Neurochemistry, Stockholm University, Stockholm, Sweden; ${ }^{3}$ Department of Pharmacology, School of Medical Sciences, University Walk, Bristol University, Bristol, UK; ${ }^{4}$ Department of Clinical Sciences South Bristol, School of Medical Sciences, University Walk, Bristol University, Bristol, UK

\begin{abstract}
The mechanisms underlying responses to drugs of abuse have been widely investigated; however, less is known about pathways normally protective against the development of drug reinforcement. These pathways are also important since they may regulate individual differences in vulnerability to addiction. The neuropeptide galanin and its binding sites are expressed in brain areas important for drug reward. Previous studies have shown that centrally infused galanin attenuates morphine place preference and peripheral injection of galnon, a galanin agonist, decreases opiate withdrawal signs. The current studies in galanin knockout (GKO) mice examined the hypothesis that galanin is an endogenous negative regulator of opiate reward and identified downstream signaling pathways regulated by galanin. We show that GKO mice demonstrate increased locomotor activation following morphine administration, which is inhibited by acute administration of galnon. GKO mice also show enhanced morphine place preference, supporting the idea that galanin normally antagonizes opiate reward. In addition, morphine-induced ERKI/2 phosphorylation was increased in the VTA of both wild-type and GKO mice, but only the GKO mice showed increases in ERKI/2 and CREB phosphorylation in the amygdala or nucleus accumbens. Furthermore, a single systemic injection of galnon in GKO mice was sufficient to reverse some of the biochemical changes brought about by morphine administration. These data suggest that galanin normally attenuates behavioral and neurochemical effects of opiates; thus, galanin agonists may represent a new class of therapeutic targets for opiate addiction.
\end{abstract}

Neuropsychopharmacology (2008) 33, 1864- 1873; doi:I0.1038/sj.npp. I 301579; published online 24 October 2007

Keywords: galanin; addiction; morphine; ERK; place preference; CREB

\section{INTRODUCTION}

Although many genetic and molecular studies have focused on molecules that predispose individuals to drug abuse, it is equally important to determine which genes and gene products may be protective against the progression from drug use to addiction. The neuropeptide galanin is an excellent candidate for an endogenous protective factor against progression to opiate abuse. Central infusion of galanin attenuates morphine place preference in the mouse (Zachariou et al, 1999). Further, galanin knockout (GKO) mice demonstrate increased signs of opiate withdrawal, whereas transgenic overexpression of galanin or administration of the galanin agonist galnon attenuates opiate withdrawal (Zachariou et al, 2003). These findings suggest that galanin has the potential

*Correspondence: Dr MR Picciotto, Department of Psychiatry, Yale University School of Medicine, 34 Park Street, 3rd Floor Research, New Haven, CT 06508, USA, Tel: + I 203737 204I, Fax: + I 203 737 2043, E-mail: marina.picciotto@yale.edu

${ }^{5}$ These authors contributed equally to this work.

Received 5 July 2007; revised 15 August 2007; accepted 15 August 2007 to decrease abuse liability of opiates. The present studies in GKO mice further explore the role of galanin in morphine reward and identify signaling pathways downstream of galanin by which galanin may act as a neuromodulator.

Galanin binds to $G$ protein-coupled receptors (GPCRs) and can modulate intracellular signaling pathways that are involved in addiction (Hawes et al, 2006a, b; Nestler, 2001; Zachariou et al, 2003). All three galanin receptor subtypes, as well as significant galanin binding, are found in the ventral tegmental area (VTA), amygdala, nucleus accumbens (NAc), cingulate cortex, and locus coeruleus (LC) (Burgevin et al, 1995; Gustafson et al, 1996; Hawes and Picciotto, 2004; Kolakowski et al, 1998; Waters and Krause, 2000), areas of the brain known to contribute to drug addiction and reward. Galanin also regulates the levels and release of a number of neurotransmitters, including dopamine, acetylcholine, and norepinephrine (Pieribone et al, 1995; Tsuda et al, 1998; Wang et al, 1999). Acute morphine administration increases locomotor activity and results in a conditioned place preference (CPP) (Suzuki et al, 1993; Wise, 1987) and these behaviors can be modulated by dopaminergic agents (Chang et al, 2004; 
Zarrindast et al, 2006a), noradrenergic depletion, or cholinergic receptor antagonists (Hikida et al, 2003; Olson et al, 2006; Rezayof et al, 2006; Zarrindast et al, 2006b). Thus, galanin may modulate the rewarding and locomotoractivating effects through modulation of multiple neurotransmitter systems.

To study whether galanin may be an endogenous protective factor able to attenuate reinforcing properties of opiates, we measured morphine-induced locomotor activity and morphine place preference in wild-type (WT) mice and GKO mice lacking the neuropeptide galanin. These studies further examined whether second messenger signaling pathways that support these morphine-regulated behaviors are modulated by galanin. At the molecular level, morphine administration leads to enhanced phosphorylation, and thus activation of extracellularregulated kinase $(\mathrm{ERK} 1 / 2)$ in the VTA, hippocampus, NAc, cingulate cortex, and amygdala (Berhow et al, 1996; Valjent et al, 2004). Increased ERK1/2 phosphorylation in the VTA is associated with morphine and psychostimulant reward (Ozaki et al, 2004) and systemic inhibition of ERK1/2 phosphorylation blocks expression of morphine and cocaine CPP (Valjent et al, 2006a). To assess galanin effects on changes in signaling, we measured the effects of morphine administration on levels of total and phosphorylated ERK1/2 in the VTA, NAc, amygdala, cingulate cortex, substantia nigra (SN), LC, caudate putamen, and hippocampus of WT and GKO mice and assessed whether the galanin receptor agonist galnon could reverse these changes.

NAc CREB has also been implicated in modulating motivational valance associated with drugs of abuse (Carlezon et al, 2005; Chartoff et al, 2003; Pandey et al, 2005). A number of pathways are known to be responsible for CREB activation (Carlezon et al, 2005; Chao and Nestler, 2004; Nestler, 2001). One second messenger pathway that leads to CREB activation is the CAMP pathway. D1 and D2 dopamine receptors, respectively, couple to stimulatory Gs proteins that via protein kinase A (PKA) enhance CREB phosphorylation and inhibitory Gi/Go proteins that inhibit cAMP production, and downstream CREB activity (Girault and Greengard, 2004). Previous work in Cath.a cells has shown that galanin administration attenuates naloxone-precipitated increases in pCREB following repeated morphine exposure, suggesting that galanin regulates CREB during opiate withdrawal (Hawes et al, $2006 \mathrm{a}, \mathrm{b})$. The purpose of the current study was to determine whether galanin might also regulate CREB signaling in vivo, using a rewarding dosing regimen of morphine. Opiate regulation of $\mathrm{Go} / \mathrm{Gq}$ pathways may also result in CREB activation (Chao et al, 2002; Widnell et al, 1996), potentially through activation of ERK signaling cascades. Therefore, we also investigated whether opiate administration leads to changes in levels of total CREB and CREB phosphorylation following morphine exposure in areas of the brain thought to support opiate reinforcement in WT and GKO animals.

These studies support the hypothesis that galanin is normally protective against the development of behaviors related to opiate addiction and further identify neuronal signaling pathways by which galanin might regulate behaviors that support addiction.

\section{MATERIALS AND METHODS}

\section{Animals}

GKO mice were generated on the 1290la/Hsd background (Wynick et al, 1998). As described previously, exons one to five of the galanin gene were replaced with a PGK-Neo cassette in reverse orientation removing the signal peptide, coding region, and the majority of the galanin-associated peptide. Galanin expression was undetectable in all tissues examined in homozygous GKO mice (Wynick et al, 1998). Once generated, the chimeras were bred to co-isogenic 1290la/Hsd WT mice. Mice used in the current study were generated by crossing GKO mice to co-isogenic WT mice of the $129 \mathrm{Ola} / \mathrm{Hsd}$ background to generate mice heterozygous for the GKO allele. These mice became the founders of the new colony. In order to generate large numbers of animals for behavioral studies, $\mathrm{KO} \times \mathrm{KO}$ and $\mathrm{WT} \times \mathrm{WT}$ offspring of HET $\times$ HET mating pairs were bred together. GKO or WT mice were then generated from several GKO $\times$ GKO or $\mathrm{WT} \times \mathrm{WT}$ mating pairs. Experimental testing occurred when mice were between the ages of 4 and 6 months. Mice were matched for age and sex for all experiments. All animal studies were conducted in accordance with guidelines from the National Institutes of Health and approved by the Yale Animal Care and Use Committee.

\section{Morphine-Induced Locomotor Activation}

Male mice (5-8 months of age) were habituated to injection and the locomotor boxes for three consecutive days. Each mouse was given two injections of $0.9 \%$ saline 15 min apart. Following the second injection, each mouse was immediately placed into a locomotor box for $40 \mathrm{~min}$. Locomotor activity was measured using a video tracking system and converted to distance traveled $(\mathrm{cm})$ using Optimax software. GKO and WT mice received a saline vehicle injection followed $15 \mathrm{~min}$ later by either $0,5,10$, or $20 \mathrm{mg} / \mathrm{kg}$ morphine in a randomized latin square design on days 4 , 6,8 , and 10. All animals received two saline injections on days $5,7,9$, and 11 . On day 12 , each mouse received an i.p. injection of $2 \mathrm{mg} / \mathrm{kg}$ galnon (synthesized as described in Saar et al, 2002) followed by an injection of $5 \mathrm{mg} / \mathrm{kg}$ morphine $15 \mathrm{~min}$ later. On day 13 , injection of $2 \mathrm{mg} / \mathrm{kg}$ galnon was followed by an injection of saline $15 \mathrm{~min}$ later. Significance was determined using repeated-measures ANOVA followed by Tukey's HSD post hoc test $(p<0.05)$.

\section{Morphine CPP}

For place preference experiments, male GKO or WT mice were used and were generated as described above. Animals were transported to the facility where behavioral training would take place at least one week prior to behavioral testing and were habituated to experimenter handling for a minimum of three days. Med Associates place conditioning boxes were modified for a nonbiased CPP paradigm. Two conditioning chambers with retractable doors were separated by a gray and white neutral chamber with a white Plexiglas floor. One conditioning chamber had a wire mesh floor and walls with black and white vertical stripes. The second conditioning chamber had a grid floor and brown marbled walls with black diagonal stripes. The location of 
each animal was recorded by photocell beam breaks and time spent in each chamber was calculated using Med-PC IV software.

During all phases of the experiment, mice were carried in their homecage into the testing room, subjects were weighed and injected, i.p., with $3.33 \mathrm{ml} / \mathrm{kg}$ of $0.9 \%$ saline vehicle or morphine sulfate $(0,0.25,3.0$, or $5.0 \mathrm{mg} / \mathrm{kg})$ just prior to placement into the CPP apparatus. Doses of morphine were selected to avoid possible ceiling effects that might preclude observation of differences between genotypes. Baseline preferences were determined prior to training. Following vehicle injection, animals were placed inside the neutral chamber and allowed to explore both conditioning chambers for $15 \mathrm{~min}$. Fourteen subjects were excluded from further testing based on a bias greater than $70 \%$ for one chamber. During the training phase of the experiment, mice received two training sessions per day over 3 days of training. During the AM session (beginning at approximately 1000 hours), mice were isolated in one conditioning chamber for $30 \mathrm{~min}$ following vehicle injection (salinepaired). During the PM session (beginning at approximately 1400 hours), animals were isolated in the opposite conditioning chamber following an injection of morphine (drug-paired). Control subjects of each genotype received saline injections $(0 \mathrm{mg} / \mathrm{kg}$ morphine) prior to placement in either of the chambers. One of the chambers was randomly designated as the drug-paired chamber for the salinetreated animals for statistical purposes. Animals were counterbalanced for drug-paired chamber according to treatment dose, genotype, and baseline preference. On the day after completion of training, animals were again injected with vehicle, placed in the neutral, center compartment of the CPP apparatus, and allowed access to both conditioning chambers for a period of $15 \mathrm{~min}$. Baseline and CPP testing sessions took place at an intermediate time between the AM and PM training sessions (approximately 1200 hours). Total time spent in each chamber during CPP testing was observed and change from baseline preference for the drug-paired chamber was used as a measure of morphine CPP.

A $2 \times 2 \times 4$ (chamber $\times$ genotype $\times$ treatment $)$ ANOVA, with conditioning chamber as a within-subject measure and genotype and treatment as between-subjects measures, was used to determine effects of galanin genotype and drug dosage on morphine CPP. Given an a priori hypothesis derived from locomotor data, post hoc one-tailed $t$-tests were performed.

A second set of locomotor experiments was performed using the $0.25 \mathrm{mg} / \mathrm{kg}$ threshold dose of morphine shown to result in morphine $\mathrm{CPP}$ in GKO but not WT mice. Male mice (6-8 months of age) received three daily $40 \mathrm{~min}$ sessions of locomotor testing. Mice received either $0.25 \mathrm{mg} /$ $\mathrm{kg}$ morphine or saline immediately prior to placement in a clear plastic locomotor box. Locomotor activity was recorded by photocells that measured the number of beam breaks and recorded as beam breaks per minute by a Dell computer using Med-PC IV software (Med Associates, St Albans, VT). On the third day, the brains were collected by rapid decapitation, and punches were taken from brain regions for biochemical analysis. Significance was determined using repeated-measures ANOVA followed by Tukey's HSD post hoc test $(p<0.05)$.

\section{Western Blot Analysis}

GKO and WT mice were administered saline or $2 \mathrm{mg} / \mathrm{kg}$ galnon $15 \mathrm{~min}$ before injection of saline or $10 \mathrm{mg} / \mathrm{kg}$ morphine. Male and female mice exactly balanced for genotype were used for western blotting studies and analyzed separately. Since no sex differences were observed, data were combined for the analyses presented here. At $5 \mathrm{~min}$ after injection, brains were harvested by rapid decapitation and tissue punches of VTA, SN, NAc, amygdala, hippocampus, LC, cingulate cortex, and caudate putamen were isolated and immediately frozen on dry ice within $10 \mathrm{~min}$ of decapitation. Cell lysis buffer $(20 \mathrm{mM}$ Hepes, $150 \mathrm{mM} \mathrm{NaCl}, 1 \mathrm{mM}$ EDTA, 1\% Triton X-100, 5\% glycerol, $10 \mathrm{mM}$ pyrophosphate, $1 \mathrm{mM} \mathrm{NaVO}_{3}, 1 \mathrm{mM}$ PMSF, $5 \mu \mathrm{g} / \mathrm{ml}$ aprotinin, and $5 \mu \mathrm{g} / \mathrm{ml}$ leupeptin, $\mathrm{pH} 7.5$, for CREB and pCREB immunoblots; $50 \mathrm{mM}$ Tris, $\mathrm{pH}$ 7.4, $1 \mathrm{mM}$ EDTA, $1 \mathrm{mM}$ EGTA $1 \%$ SDS, $1 \mathrm{mM}$ PMSF for ERK and pERK immunoblots) was added to each frozen tissue punch and immediately pulse sonicated for $5 \mathrm{~s}$, then cleared by centrifugation for $15 \mathrm{~min}$ at $4{ }^{\circ} \mathrm{C}$. Lowry reagents (Biorad, Hercules, CA) were used to determine protein concentrations according to the manufacturer's instructions. In total, $5-10 \mu \mathrm{g}$ of protein for each sample was separated on 8.5 or $10 \%$ poly-acrylamide gels by SDS-PAGE and transferred to nitrocellulose membranes. Blots were blocked in 5\% BSA/ TBS or $5 \%$ Milk/TBS-T for $1 \mathrm{~h}$, then incubated overnight at $4^{\circ} \mathrm{C}$ in primary antibody. Polyclonal antisera specific for ERK1/2, and polyclonal or monoclonal antisera specific for the phosphorylated form of ERK (Cell Signaling, Beverley, MA) were used at a dilution of $1: 1000$ and $1: 2000$, respectively. Polyclonal antisera specific for $\mathrm{CREB}$ or phosphorylated serine 133 on CREB (Cell Signaling, Beverley, MA) were used at dilutions of $1: 1000$. TH immunoreactivity was used to verify the accuracy and consistency of VTA tissue punches. Anti-TH polyclonal antibody (Chemicon, Temecula, CA) was used at a dilution of 1:2000. Glyceraldehyde-3-phosphate dehydrogenase (GAPDH) immunoreactivity was used as an internal standard, by incubating the blots either alone for $30 \mathrm{~min}$ at room temperature in anti-GAPDH monoclonal primary antibody (Advanced ImmunoChemical Inc., Long Beach, CA) diluted 1:2000 in 5\% BSA/TBS following the overnight incubation with CREB, or TH primary antibodies, or overnight in a cocktail with the ERK or pERK antibodies. Blots were washed three times for 5 min with TBS ( $\mathrm{pH} 7.4)$ / $0.05 \%$ Tween (TBST), incubated with peroxidase-labeled anti-rabbit IgG 1:2000 (Vector Laboratories, Inc., Burlingame, CA), or IR Dye 800-conjugated anti-rabbit IgG (Rockland Inc., Gilbertsville, PA) and Alexa fluor 680conjugated anti-mouse IgG (Molecular Probes, Eugene, OR) secondary antibodies for $1 \mathrm{~h}$ at room temperature and washed five times with TBS-T. Bands were visualized by enhanced chemiluminescence and exposure to Kodak Biomax $\mathrm{MR}$ or $\mathrm{AR}$ film. Immunoblots incubated with fluorescent-conjugated secondary antibodies were scanned using the LI-COR Odyssey imager (LI-COR Biosciences, Lincoln, NE). Protein loading was verified using Ponceau staining and GAPDH immunoblotting. Bands were quantified using NIH Image software (URL:http//rsb.info.nig.gov/ nih-image), or the odyssey imaging software. Levels of protein phosphorylation were determined by calculating the 
ratio of phosphorylated band intensity to total protein band intensity. Data were normalized to the WT saline group to allow comparison across multiple blots by dividing each value by the average of the WT saline group and multiplying by 100 . Significance was determined using ANOVA followed by the least significant difference post hoc test $(p<0.05)$.

\section{RESULTS}

\section{Morphine-Induced Locomotor Activity and CPP}

Most drugs of abuse, including morphine, can increase locomotion when injected acutely (Wise, 1987). We therefore used locomotor activation as a behavioral measure of sensitivity to opiates in mice lacking the neuropeptide galanin and their WT controls $(\mathrm{F}(7,97)=9.082, p<0.05$; Figure 1a). WT mice of the 1290la/Hsd strain did not show a significant increase in locomotor activity following morphine injection at the doses we used (Figure 1a). Morphine can both increase and depress locomotor activity in different strains of mice (Eidelberg and Erspamer, 1975; Oliverio et al, 1975), and the lack of locomotor activation in WT mice in the current study may represent a strain difference between 1290la/Hsd mice and other strains, such as $\mathrm{C} 57 \mathrm{BL} / 6$ mice, that are more susceptible to the locomotor-activating effects of morphine. In contrast, GKO mice on the 1290la/Hsd background showed a significant increase in locomotor activity following morphine injection as compared to saline-injected controls $(p<0.05$, Tukey HSD post hoc test; Figure 1a).


Figure I Locomotor activity following acute morphine administration. (a) Galanin wild-type (WT; $n=10$ ) and knockout (GKO; $n=11$ ) mice were administered an acute i.p. injection of saline followed by 0,5 , 10 , or $20 \mathrm{mg} / \mathrm{kg}$ morphine $15 \mathrm{~min}$ later. Locomotor activity was significantly increased in GKO animals as compared to WT following acute morphine administration. Significance was determined using repeated-measures ANOVA followed by Tukey's HSD post hoc test $(F(7,97)=9.082$, $p<0.05)$. (b) I.p. injection of $2 \mathrm{mg} / \mathrm{kg}$ galnon $15 \mathrm{~min}$ prior to morphine administration $(5 \mathrm{mg} / \mathrm{kg}$ ) significantly inhibited morphine-induced increases in locomotor activity of GKO animals. Knockout animals receiving $5 \mathrm{mg} / \mathrm{kg}$ morphine are the only group significantly different from baseline (*). Significance was determined using repeated-measures ANOVA followed by Tukey's HSD post hoc test $(F(7,99)=6.153, p<0.05)$.
To further determine whether galanin signaling inhibits morphine-induced locomotor activity directly, galanin WT and GKO mice were treated with galnon, a galanin receptor agonist. Galnon, 7-((9-fluorenylmethoxycarbonyl)cyclohexylalanyllysyl)amino-4-methylcoumarin, is a low molecular weight, non-peptide galanin receptor agonist that displaces $\left[{ }^{125} \mathrm{I}\right]$ glanin from its binding sites and inhibits adenylyl cyclase activity through activation of galanin receptors (Saar et al, 2002; Wu et al, 2003). Galnon administration reversed the morphine-induced increase in locomotor activation seen in GKO mice $(F(7,99)=6.513, p<0.05$; Figure 1b); however, galnon did not further reduce locomotor activity in WT mice and had no effect on its own ( $p>0.05$, Tukey HSD post hoc test).

CPP for morphine was evaluated in GKO and WT mice based on an earlier finding that infusion of galanin into the brain attenuates morphine place preference in WT C57BL/6 mice (Zachariou et al, 1999). Consistent with the idea that galanin normally antagonizes opiate reward, GKO mice show increased sensitivity to morphine place preference compared to WT mice. There was a significant interaction of genotype and drug treatment $\mathrm{F}(3,150)=3.378, p<0.05)$. Post hoc $t$-tests showed that at the $0.25 \mathrm{mg} / \mathrm{kg}$ dose of morphine, GKO subjects showed a greater increase in preference for the drug-paired chamber than WT animals as measured by a significantly larger change from baseline $(p<0.05$; Figure $2 \mathrm{a})$. There was no significant difference in change of preference for the drug-paired chamber between WT and GKO subjects. There was also no effect of treatment or genotype on baseline preference for the conditioning chambers (Figure $2 \mathrm{~b}$ ). At the threshold dose for morphine place preference $(0.25 \mathrm{mg} / \mathrm{kg})$, neither WT nor GKO mice showed locomotor activation (Figure 2d).

\section{Neurochemical Changes Downstream of Morphine Signaling}

Galanin modulates the release of a number of neurotransmitters in brain areas involved in behavioral responses to drugs of abuse (Pieribone et al, 1995; Tsuda et al, 1998; Wang et al, 1999). We therefore evaluated the levels and activity of signaling molecules known to be activated by drugs of abuse in tissue punches from regions involved in modulation of drug reward, including the VTA and catecholaminergic projection areas. We measured changes in total levels or phosphorylation state of CREB and ERK1/2 in GKO mice to identify galanin-associated neurochemical changes resulting from acute morphine treatment.

At a dose of $10 \mathrm{mg} / \mathrm{kg}$, morphine induced a significant increase in the phosphorylation state of ERK1/2 (WT-Sal vs WT-Mor: $\mathrm{F}(3,19)=6.09$; $p<0.01$; WT-Sal vs KO-Mor: $\mathrm{F}(3,20)=22.8 ; p<0.01$; Tukey HSD post hoc test; Figure $3 \mathrm{a})$, but not CREB (WT-Sal vs WT-Mor: $\mathrm{F}(3,19)=2.96 ; p>0.05$; WT-Sal vs. KO-Mor: $\mathrm{F}(3,21)=2.9 ; p>0.5$; Tukey HSD post hoc test; Figure $3 \mathrm{~b}$ ), in the VTA of both galanin WT and GKO animals. No changes in total ERK and CREB were observed at any dose and no changes in P-ERK or P-CREB were observed in animals of either genotype treated with $0.25 \mathrm{mg} / \mathrm{kg}$ morphine (not shown). Since inhibition of ERK1/2 phosphorylation in the VTA blocks the rewarding effects of morphine (Berhow et al, 1996; Ozaki et al, 2004), the observed increases in ERK1/2 activation may regulate 



c

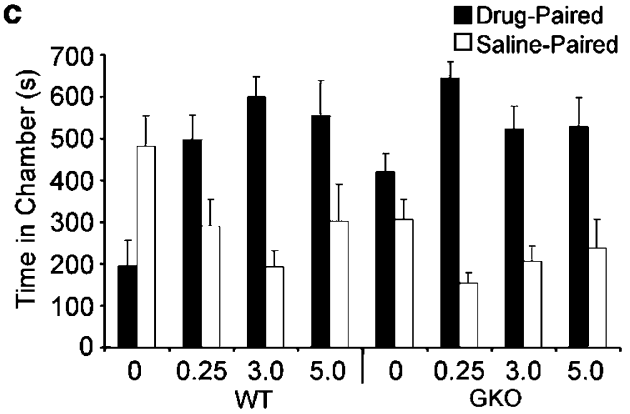

d

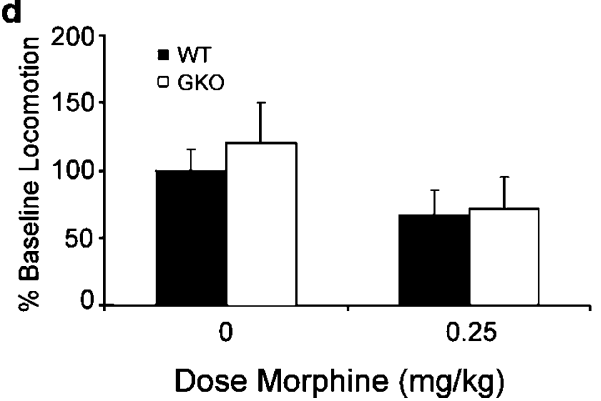

Figure 2 Morphine place preference. Morphine conditioned place preference (CPP) in wild-type (WT) and galanin knockout (GKO) mice. (a) Change from baseline preference for the drug-paired chamber is shown for GKO and WT mice. GKO mice showed significantly greater morphine preference than WT mice at the $0.25 \mathrm{mg} / \mathrm{kg}$ dose of morphine (*p $<0.05$ ). (b) Baseline levels of total time spent in the drug-paired and saline-paired chambers. (c) Total time spent in the drug-paired and saline-paired chambers is shown for mice after CPP training. Control animals of each genotype $(0 \mathrm{mg} / \mathrm{kg})$ received saline vehicle prior to placement in either chamber, with one chamber randomly designated the 'drug-paired' chamber for statistical analysis. (d) Galanin WT( $n=17)$ and $\mathrm{GKO}(n=15)$ mice were administered an acute i.p. injection of saline or $0.25 \mathrm{mg} / \mathrm{kg}$ morphine. The total locomotor activity measured as beam breaks were averaged over three days. There were no significant differences in locomotor activity between genotypes that received either treatment. Error bars represent standard error about the mean.

morphine reward. A trend for slightly higher levels of ERK1/2 phosphorylation in GKO mice (WT-Mor vs KO-Mor: $\mathrm{F}(3,19)=2.4 ; p=0.08)$ correlates with the ten-
Figure 3 Changes in levels and phosphorylation state of CREB and ERK in the VTA following administration of acute morphine alone or galnon prior to morphine. Galanin wild-type and knockout mice were administered an acute i.p. injection of $10 \mathrm{mg} / \mathrm{kg}$ morphine alone or were administered morphine $15 \mathrm{~min}$ after an i.p. injection of galnon at a dose of $2 \mathrm{mg} / \mathrm{kg}$ and killed $5 \mathrm{~min}$ after the morphine injection. (a, b) Tissue punches from the ventral tegmental area (VTA) were immunoblotted for ERK and phospho-ERK and CREB and phospho-CREB. ${ }^{*} p$-value $<0.05$ with respect to saline-treated WT controls. WT-Saline, $n=4$; GKO-Saline, $n=4 ; \quad$ WT-Morphine, $n=8 ; \quad$ GKO-Morphine, $n=8 ; \quad$ WT-Galnon + Morphine, $n=8$; GKO-Galnon + Morphine, $n=9$.

dency for these animals to show increased behavioral sensitivity to morphine. Together with studies showing that ERK activity is necessary for place preference (Girault et al, 2007; Salzmann et al, 2003; Valjent et al, 2001, 2006a,b, 2004) and that galanin reduces morphine CPP (Zachariou et al, 1999), these data suggest that galanin might regulate morphine reward via regulation of ERK signaling in the VTA. It is also possible that ERK activity in the VTA regulates the ability of galanin to modulate morphineinduced locomotor activation.

To identify changes in intracellular signaling that regulate locomotor behavior, we evaluated the ability of galnon to reverse neurochemical changes resulting from morphine treatment in GKO mice. ERK activation in the VTA of galnon-injected animals was significantly lower than animals that received morphine alone (KO-Mor $v s \mathrm{KO}-\mathrm{Gal}$ + Mor: $\mathrm{F}(3,21)=5.8 ; p<0.01$; Tukey HSD post hoc test; Figure $3 \mathrm{a}$ ); therefore, galnon attenuated the morphineinduced ERK activation in the VTA of both WT and GKO animals.

ERK phosphorylation in the NAc in response to treatment with drugs of abuse is thought to be important for locomotor activation and reward (Valjent et al, 2004). Consistent with the propensity for GKO mice to show morphine-induced locomotor activation and an augmentation of morphine CPP, levels of phosphorylated ERK1/2 were significantly increased in the NAc of GKO mice (WTSal $v s$ KO-Mor: $p<0.01 ; \mathrm{F}(3,19)=9.56$; Tukey HSD post hoc test) but not in WT mice following morphine exposure (WT-Sal vs WT-Mor: $p>0.05 ; \mathrm{F}(3,19)=1.88$; Tukey HSD 
post hoc test; Figure 4a). The lack of morphine-induced ERK1/2 phosphorylation in the NAc of WT mice might explain the absence of morphine-induced locomotor activation in mice of the 1290la/Hsd strain. Total ERK, total CREB, and CREB phosphorylation levels were all unchanged in the NAc of WT and GKO mice following morphine administration (Figure $4 \mathrm{a}$ and $\mathrm{b}$ ).

In the amygdala, both WT and GKO mice treated with $10 \mathrm{mg} / \mathrm{kg}$ morphine exhibited a significant increase in ERK1/2 phosphorylation (WT-Sal vs WT-Mor: $\mathrm{F}(3,21)$ $=6.56 ; \quad p<0.01 ; \quad$ WT-Sal vs KO-Mor: $\mathrm{F}(3,20)=9.6$; $p<0.01$; Tukey HSD post hoc test; Figure 5a) that was reversed by systemic galnon administration prior to morphine injection. In contrast, CREB phosphorylation was significantly increased by $150 \%$ in the amygdala of GKO mice (WT-Sal vs KO-Mor: $\mathrm{F}(3,20)=19.67 ; p<0.01$; Tukey HSD post hoc test), but not regulated in WT mice following acute morphine injection (WT-Sal vs WT-Mor: $\mathrm{F}(3,20)$ $=1.9 ; p>0.05$; Tukey HSD post hoc test; Figure 5b). Furthermore, a single injection of galnon did not attenuate CREB phosphorylation in the amygdala of GKO mice. At a threshold dose of $0.25 \mathrm{mg} / \mathrm{kg}$ morphine, there was no CREB activation in the amygdala of WT or GKO mice (Figure 5c).

There were no significant changes in levels of total or phosphorylated ERK or CREB in the SN, hippocampus, LC, caudate putamen, or cingulate cortex after acute morphine treatment in either genotype $(F ' s<1.0)$ (data not shown).


Figure 4 Changes in levels and phosphorylation state of CREB and ERK in the NAc following administration of acute morphine alone or galnon prior to morphine administration. Galanin wild-type and knockout mice were administered an acute i.p. injection of $10 \mathrm{mg} / \mathrm{kg}$ morphine alone or were administered morphine $15 \mathrm{~min}$ after an i.p. injection of galnon at a dose of $2 \mathrm{mg} / \mathrm{kg}$ and decapitated $5 \mathrm{~min}$ after the morphine injection. $(\mathrm{a}, \mathrm{b})$ Tissue punches from the nucleus accumbens (NAc) were immunoblotted for ERK and phospho-ERK and CREB and phospho-CREB. * $p$-value $<0.05$ with respect to saline-treated WT controls. WT-Saline, $n=4$; GKO-Saline, $n=4 ; \quad$ WT-Morphine, $n=8 ; \quad$ GKO-Morphine, $n=8$, WT-Galnon + Morphine, $n=8$; GKO-Galnon + Morphine- $n=9$.

\section{DISCUSSION}

Galanin null mutant mice were more sensitive to morphine than their WT counterparts in tests of locomotor activation and morphine CPP. GKO mice, but not WT animals exhibited increased locomotor activation following acute morphine treatment. Galnon coadministration reversed the morphine-induced increase in locomotor activity, suggesting that morphine locomotor activation in GKO mice is not due to a developmental difference in these animals, but that galanin acutely inhibits the psychomotor stimulant properties of morphine in adulthood. Morphine place preference was also enhanced in GKO mice compared to WT controls, supporting neurochemical data suggesting that galanin counters drug reward (Zachariou et al, 1999). GKO mice also showed morphine-dependent increases in pERK in the NAc and PCREB in the amygdala, areas of the brain that are
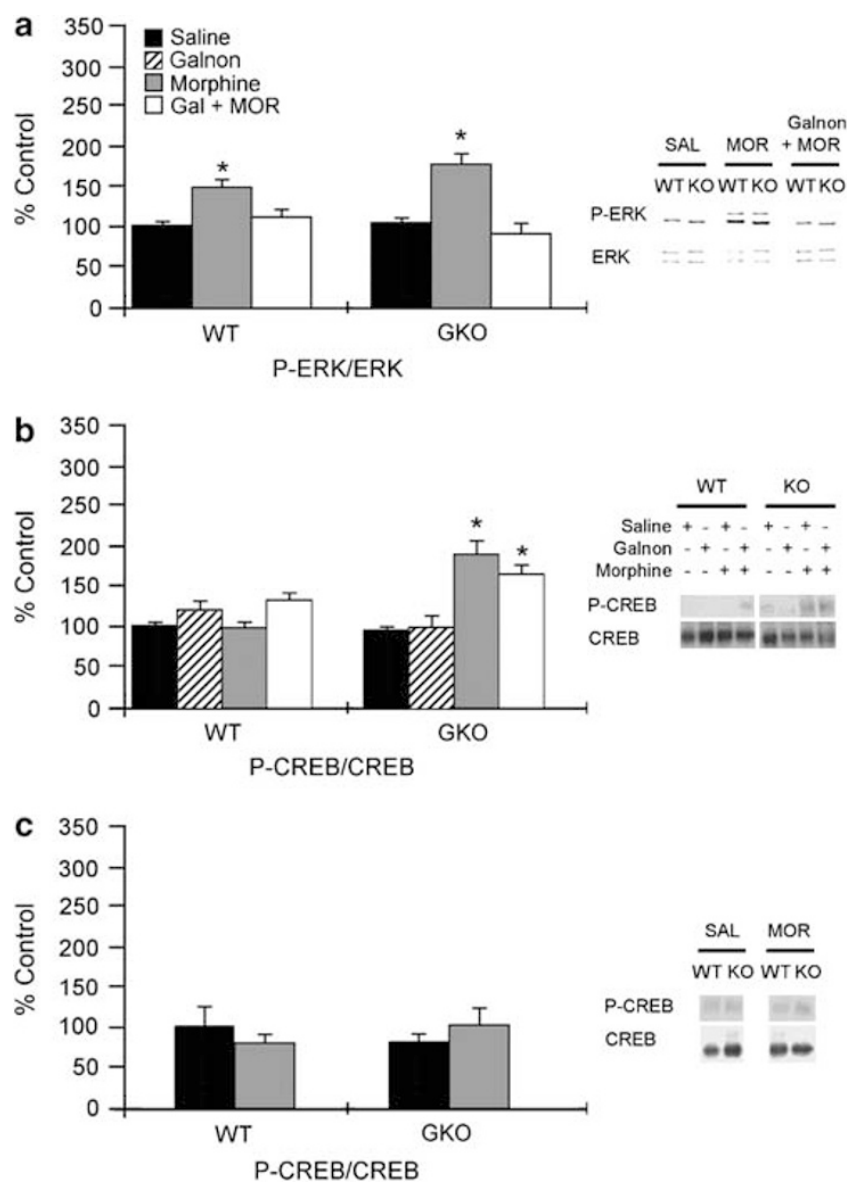

Figure 5 Changes in levels and phosphorylation state of CREB and ERK in the amygdala following administration of acute morphine alone or galnon prior to morphine administration. $(a, b)$ Tissue punches from the amygdala were immunoblotted for CREB and phospho-CREB and ERK and phosphoERK in galanin wild-type and knockout mice administered an acute i.p. injection of $10 \mathrm{mg} / \mathrm{kg}$ morphine alone or administered morphine $15 \mathrm{~min}$ after an i.p. injection of $2 \mathrm{mg} / \mathrm{kg}$ galnon and killed $5 \mathrm{~min}$ after the morphine injection. (c) CREB and phospho-CREB signaling in mice injected with $0.25 \mathrm{mg} / \mathrm{kg}$ morphine, assessed for locomotor activation on three successive days, and killed on the third day 5 min after morphine injection, immediately after the last locomotor test; $*$-value $<0.05$ with respect to saline-treated WT controls. WT-Saline, $n=4$; GKO-Saline, $n=3$; WTMorphine, $n=8$; GKO-Morphine, $n=8 ;$ WT-Galnon + Morphine, $n=8$; GKO-Galnon + Morphine, $n=9$. 


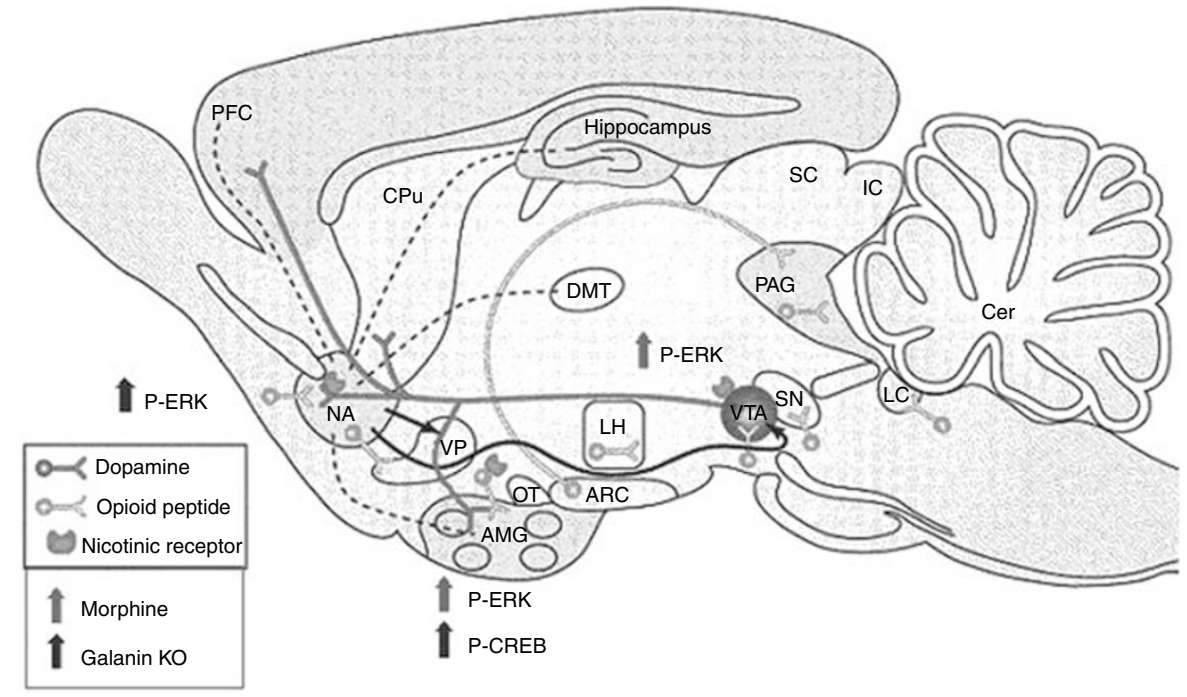

Figure 6 Summary of neurochemical changes. Significant changes in ERK and CREB phosphorylation that are due to morphine and independent of galanin are indicated in gray. Significant neurochemical changes that are induced by morphine and are altered in galanin knockout mice, and thus likely to be modulated by galanin, are indicated in black. Ventral tegmental area (VTA), nucleus accumbens (NAc), amygdala (AMG), ventral portion of the caudateputamen (CPu), substantia nigra (SN), prefrontal cortex (PFC), and locus coeruleus (LC).

critical for behavioral effects of morphine (Figure 6). In the VTA and amygdala, both WT and GKO mice showed elevated levels of pERK that were reversible by galnon, but this effect was exaggerated in the VTA of GKO mice. Morphine-dependent increases in levels of pERK but not pCREB were reversible by systemic administration of galnon, suggesting that galanin acutely regulates changes in ERK signaling in the VTA, NAc, and amygdala. There was no effect of morphine on ERK or CREB signaling in the SN, hippocampus, LC, cingulate cortex, or caudate putamen, demonstrating that morphine-associated effects on ERK and CREB signaling were specific to the VTA, NAc, and amygdala in these studies. These data support the hypothesis that galanin antagonizes opiate reinforcement and reward and identify ERK signaling in the VTA, NAc, and amygdala as a potential downstream target for galanin effects on opiate-dependent behavior.

Galanin, opioid, and DA receptors are GPCRs that modulate many signaling pathways including those involving ERK1/2 (Belcheva et al, 2005, 2001; Hawes et al, 2006a; Loh and Smith, 1990; Williams et al, 2001). Chronic morphine exposure (Berhow et al, 1996) and stimulation of several GPCRs, including opioid receptors, leads to ERK activation in the VTA (Eitan et al, 2003) and inhibition of ERK activity in the VTA suppresses the rewarding effects of morphine (Ozaki et al, 2004). The current data support these findings, showing significant morphine-CPP and increases in phosphorylation of ERK1/2 in the VTA of both galanin WT and GKO mice following acute administration of a rewarding dose of morphine. These data collected $5 \mathrm{~min}$ following $10 \mathrm{mg} / \mathrm{kg}$ morphine injection differ somewhat from earlier findings (Berhow et al, 1996) that reported no changes in ERK activation in the VTA $2 \mathrm{~h}$ following a single injection of a higher dose of morphine, however they are consistent with data showing that ERK phosphorylation peaks approximately $5 \mathrm{~min}$ after drug administration (Valjent et al, 2004). Similarly, we observed no change in
ERK signaling in the hippocampus or cingulate cortex at a 5 min time point, likely due to the fact that activation of ERK signaling by morphine in these areas peaks at $20 \mathrm{~min}$ post injection (Valjent et al, 2004). Other studies show that inhibition of ERK during place conditioning blocks cocaine CPP (Valjent et al, 2006a). ERK induction at the $5 \mathrm{~min}$ time point indicates that stimulation of ERK would occur during exposure to the training chamber during place conditioning. In addition to the finding that GKO mice showed enhanced morphine CPP at low doses of morphine, there was a tendency for GKO mice to show elevated levels of phosphorylated ERK in the VTA in comparison to their WT counterparts, suggesting that galanin-associated regulation of ERK may modulate behavioral sensitivity to morphine.

Effects of morphine on ERK signaling and locomotor activation were reversed by the galanin agonist, galnon. Galnon only partially reversed morphine-induced elevations of phosphorylated ERK in the VTA of GKO and WT mice, suggesting that galanin exerts limited control over ERK signaling in the VTA. By contrast, in the NAc, a single galnon injection completely abolished morphine-induced ERK activation. Galnon is a galanin agonist that is thought to have some selectivity for GalR1 over GalR2 and GalR3 (Saar et al, 2002; Wu et al, 2003). Galnon can act as a galanin receptor agonist in vivo in behavioral paradigms modulated by the endogenous peptide galanin, including opiate withdrawal (Zachariou et al, 2003), feeding behavior (Abramov et al, 2004), seizures (Saar et al, 2002), and anxiety (Rajarao et al, 2007). However, it should be noted that galnon can also interact directly with some $G$ proteins (Flören et al, 2005), thus the action of galnon may not be limited to activation of GalR1. GalR1 is negatively coupled to adenylyl cyclase and activation of this receptor subtype can therefore decrease PKA-mediated signaling as well as neuronal excitability (Berthold et al, 1997). Thus, galnonmediated activation of GalR1 could reverse the effects of morphine on ERK signaling either by decreasing the 
excitability of neurons in which these signaling pathways were activated, or by decreasing the contribution of cyclicAMP-mediated pathways in those neurons through activation of Gi. Interestingly, morphine-associated NAc activation of ERK and locomotor activation were specific to GKO mice. Unlike other strains that show robust morphinedependent locomotor activation, the 129Ola/Hsd mice in these studies did not display locomotor hyperactivity in response to morphine administration. GKO animals on the $129 \mathrm{Ola} /$ Hsd background, however, showed robust locomotor activation in response to morphine. Together, these data suggest that galanin is a negative modulator of the behavioral and neurochemical signs of acute opiate administration in vivo.

Galanin may modulate responses to drugs of abuse through multiple transmitter systems, either independently or in combination, in the brain regions involved in these behaviors. DA release in the NAc is thought to be critical for locomotor activation and opiate reward (Di Chiara and Imperato, 1988). Galanin decreases presynaptic DA release in striatal slices (Tsuda et al, 1998) and one possible explanation for the heightened sensitivity of GKO mice to morphine-induced locomotion and CPP is that knockout of the peptide may result in hyperactivation of DA signaling following opiate administration. Whereas mRNA levels for galanin receptors are low in the NAc, galanin receptor binding is quite prominent (Burgevin et al, 1995; Gustafson et al, 1996; Hawes and Picciotto, 2004; Kolakowski et al, 1998; Waters and Krause, 2000), indicating that localization of galanin receptors on DA terminals might regulate DA release. Galanin is also known to be a potent inhibitory modulator of basal acetylcholine (ACh) release in the striatum (Antoniou et al, 1997) and it has been demonstrated that M1-muscarinic receptors and high-affinity nicotinic receptors regulate the secondary rewarding effects of cues paired with a primary reinforcer such as morphine (Brunzell et al, 2006; Carrigan and Dykstra, 2007). Lack of galanin may therefore increase availability of $\mathrm{ACh}$, thus increasing the sensitivity of GKO mice to opiates. Finally, galanin has also been shown to decrease GABA release in the cortex and striatum (Antoniou et al, 1997; Ellis and Davies, 1994), potentially increasing excitability in these brain areas and contributing to morphine's behavioral effects. The effects of galanin on morphine-related behaviors may therefore modulate reward pathways through multiple neurotransmitter systems.

A dramatic increase in CREB phosphorylation was detected in the amygdala of GKO mice treated with $10 \mathrm{mg} /$ $\mathrm{kg}$ morphine, but not WT mice. CREB is phosphorylated and activated in the amygdala by several drugs of abuse including opiates (Konradi et al, 1994; Olson et al, 2006; Shaw-Lutchman et al, 2002). In addition, opiate withdrawal induced by naloxone can also increase CREB phosphorylation in cultured neurons and in vivo (Chartoff et al, 2003), an effect that is abolished by coadministration of galanin (Hawes et al, 2006b). The behavioral implications of the increased CREB phosphorylation in the amygdala in GKO mice are unclear since, unlike morphine-induced locomotor activation and ERK phosphorylation, which were reversed by galnon administration in GKO mice, CREB activation in the amygdala was not reversed following a single injection of galnon. It is possible that lack of galanin during development may result in changes in the amygdala that make CREB activation in this brain area more sensitive to opiate administration. Alternatively, it is possible that galanin is important for drug-associated learning that occurs during early exposure to morphine. Galanin can block memory consolidation processes (Kinney et al, 2003) and the amygdala is critical for cue-associated learning (Robbins and Everitt, 1996). A low dose of morphine that resulted in morphine CPP did not affect phosphorylation of CREB in GKO mice, however. Thus, although activation of CREB in the amygdala may contribute to drug-associated learning, it is not essential for associating contextual cues with reward.

The data presented here provide strong evidence demonstrating that galanin normally acts as a negative regulator of the acute behavioral response to morphine. In addition, together with evidence indicating that phosphorylation of ERK is necessary for drug reinforcement (Ozaki et al, 2004; Valjent et al, 2001, 2006a, b, 2004, 2005), these data suggest that galanin may modulate drug-dependent behaviors via activation of ERK in brain areas such as the VTA, NAc, and amygdala. Thus, galanin agonists may prove useful in attenuating the rewarding properties of opiates.

\section{ACKNOWLEDGEMENTS}

This work was supported by grants DA15425 and DA00436 from the National Institutes of Health.

\section{DISCLOSURE/CONFLICT OF INTEREST}

The authors declare that over the last 3 years MRP has received compensation from Pfizer for scientific consultation unrelated to the present work. None of the other authors has any conflicts of interest.

\section{REFERENCES}

Abramov U, Florén A, Echevarria DJ, Brewer A, Manuzon H, Robinson JK et al (2004). Regulation of feeding by galnon. Neuropeptides 38: 55-61.

Antoniou K, Kehr J, Snitt K, Ogren SO (1997). Differential effects of the neuropeptide galanin on striatal acetylcholine release in anaesthetized and awake rats. Br J Pharmacol 121: 1180-1186.

Belcheva MM, Clark AL, Haas PD, Serna JS, Hahn JW, Kiss A et al (2005). $\mathrm{Mu}$ and kappa opioid receptors activate ERK/MAPK via different protein kinase $C$ isoforms and secondary messengers in astrocytes. J Biol Chem 280: 27662-27669.

Belcheva MM, Szucs M, Wang D, Sadee W, Coscia CJ (2001). $\mathrm{Mu}$-opioid receptor-mediated ERK activation involves calmodulin-dependent epidermal growth factor receptor transactivation. J Biol Chem 276: 33847-33853.

Berhow MT, Hiroi N, Nestler EJ (1996). Regulation of ERK (extracellular signal regulated kinase), part of the neurotrophin signal transduction cascade, in the rat mesolimbic dopamine system by chronic exposure to morphine or cocaine. J Neurosci 16: 4707-4715.

Berthold M, Kahl U, Jureus A, Kask K, Nordvall G, Langel Ü et al (1997). Mutagenesis and ligand modification studies on galanin binding to its GTP-binding-protein-coupled receptor GalR1. Eur J Biochem 249: 601-606.

Brunzell DH, Chang JR, Schneider B, Olausson P, Taylor JR, Picciotto MR (2006). Beta2-subunit-containing nicotinic 
acetylcholine receptors are involved in nicotine-induced increases in conditioned reinforcement but not progressive ratio responding for food in C57BL/6 mice. Psychopharmacology 184: 328-338.

Burgevin MC, Loquet I, Quarteronet D, Habert-Ortoli E (1995). Cloning, pharmacological characterization, and anatomical distribution of a rat cDNA encoding for a galanin receptor. J Mol Neurosci 6: 33-41.

Carlezon Jr WA, Duman RS, Nestler EJ (2005). The many faces of CREB. Trends Neurosci 28: 436-445.

Carrigan KA, Dykstra LA (2007). Behavioral effects of morphine and cocaine in M1 muscarinic acetylcholine receptor-deficient mice. Psychopharmacology (Berl) 191: 985-993.

Chang CK, Wang NL, Lin MT (2004). Inhibition of the dopamine system in rat amygdala attenuates the picrotoxin-induced locomotor hyperactivity and hypertension. Clin Exp Pharmacol Physiol 31: 284-288.

Chao J, Nestler EJ (2004). Molecular neurobiology of drug addiction. Annu Rev Med 55: 113-132.

Chao JR, Ni YG, Bolanos CA, Rahman Z, DiLeone RJ, Nestler EJ (2002). Characterization of the mouse adenylyl cyclase type VIII gene promoter: regulation by CAMP and CREB. Eur J Neurosci 16: 1284-1294.

Chartoff EH, Papadopoulou M, Konradi C, Carlezon Jr WA (2003). Dopamine-dependent increases in phosphorylation of cAMP response element binding protein (CREB) during precipitated morphine withdrawal in primary cultures of rat striatum. J Neurochem 87: 107-118.

Di Chiara G, Imperato A (1988). Drugs abused by humans preferentially increase synaptic dopamine concentrations in the mesolimbic system of freely moving rats. Proc Natl Acad Sci USA 85: 5274-5278.

Eidelberg E, Erspamer R (1975). Dopaminergic mechanisms of opiate actions in brain. J Pharmacol Exp Ther 192: 50-57.

Eitan S, Bryant CD, Saliminejad N, Yang YC, Vojdani E, Keith Jr D et al (2003). Brain region-specific mechanisms for acute morphine-induced mitogen-activated protein kinase modulation and distinct patterns of activation during analgesic tolerance and locomotor sensitization. J Neurosci 23: 8360-8369.

Ellis Y, Davies JA (1994). The effect of neuropeptides on the release of neurotransmitter amino acids from rat striatum. Neuropeptides 26: 65-69.

Flören A, Sollenberg U, Lundstrom L, Zorko M, Stojan J, Budihna $M$ et al (2005). Multiple interaction sites of galnon trigger its biological effects. Neuropeptides 39: 547-558.

Girault JA, Greengard P (2004). The neurobiology of dopamine signaling. Arch Neurol 61: 641-644.

Girault JA, Valjent E, Caboche J, Herve D (2007). ERK2: a logical AND gate critical for drug-induced plasticity? Curr Opin Pharmacol 7: 77-85.

Gustafson EL, Smith KE, Durkin MM, Gerald C, Branchek TA (1996). Distribution of a rat galanin receptor mRNA in rat brain. NeuroReport 7: 953-957.

Hawes JJ, Narasimhaiah R, Picciotto MR (2006a). Galanin and galanin-related peptide modulate neurite outgrowth via PKCmediated activation of ERK. Eur J Neurosci 23: 2937-2946.

Hawes JJ, Narasimhaiah R, Picciotto MR (2006b). Galanin attenuates cyclic AMP regulatory element-binding protein (CREB) phosphorylation induced by chronic morphine and naloxone challenge in Cath.a cells and primary striatal cultures. I Neurochem 96: 1160-1168.

Hawes JJ, Picciotto MR (2004). Characterization of GalR1, GalR2, and GalR3 immunoreactivity in catecholaminergic nuclei of the mouse brain. J Comp Neurol 479: 410-423.

Hikida T, Kitabatake Y, Pastan I, Nakanishi S (2003). Acetylcholine enhancement in the nucleus accumbens prevents addictive behaviors of cocaine and morphine. Proc Natl Acad Sci USA 100: 6169-6173.
Kinney JW, Starosta G, Crawley JN (2003). Central galanin administration blocks consolidation of spatial learning. Neurobiol Learn Mem 80: 42-54.

Kolakowski Jr LF, O'Neill GP, Howard AD, Broussard SR, Sullivan KA, Feighner SD et al (1998). Molecular characterization and expression of cloned human galanin receptors GALR2 and GALR3. J Neurochem 71: 2239-2251.

Konradi C, Cole RL, Heckers S, Hyman SE (1994). Amphetamine regulates gene expression in rat striatum via transcription factor CREB. J Neurosci 14: 5623-5634.

Loh HH, Smith AP (1990). Molecular characterization of opioid receptors. Annu Rev Pharmacol Toxicol 30: 123-147.

Nestler EJ (2001). Molecular neurobiology of addiction. Am J Addict 10: 201-217.

Oliverio A, Castellano C, Eleftheriou BE (1975). Morphine sensitivity and tolerance: a genetic investigation in the mouse. Psychopharmacologia 42: 219-224.

Olson VG, Heusner CL, Bland RJ, During MJ, Weinshenker D, Palmiter RD (2006). Role of noradrenergic signaling by the nucleus tractus solitarius in mediating opiate reward. Science 311: 1017-1020.

Ozaki S, Narita M, Narita M, Ozaki M, Khotib J, Suzuki T (2004). Role of extracellular signal-regulated kinase in the ventral tegmental area in the suppression of the morphine-induced rewarding effect in mice with sciatic nerve ligation. J Neurochem 88: 1389-1397.

Pandey SC, Chartoff EH, Carlezon Jr WA, Zou J, Zhang H, Kreibich AS et al (2005). CREB gene transcription factors: role in molecular mechanisms of alcohol and drug addiction. Alcohol Clin Exp Res 29: 176-184.

Pieribone VA, Xu ZQ, Zhang X, Grillner S, Bartfai T, Hökfelt T (1995). Galanin induces a hyperpolarization of norepinephrinecontaining locus coeruleus neurons in the brainstem slice. Neuroscience 64: 861-874.

Rajarao SJ, Platt B, Sukoff SJ, Lin Q, Bender CN, Nieuwenhuijsen BW et al (2007). Anxiolytic-like activity of the non-selective galanin receptor agonist, galnon. Neuropeptides 41: 307-320.

Rezayof A, Zatali H, Haeri-Rohani A, Zarrindast MR (2006). Dorsal hippocampal muscarinic and nicotinic receptors are involved in mediating morphine reward. Behav Brain Res 166: 281-290.

Robbins TW, Everitt BJ (1996). Neurobehavioural mechanisms of reward and motivation. Curr Opin Neurobiol 6: 228-236.

Saar K, Mazarati AM, Mahlapuu R, Hallnemo G, Soomets U, Kilk K et al (2002). Anticonvulsant activity of a nonpeptide galanin receptor agonist. Proc Natl Acad Sci USA 99: 7136-7141.

Salzmann J, Marie-Claire C, Le Guen S, Roques BP, Noble F (2003). Importance of ERK activation in behavioral and biochemical effects induced by MDMA in mice. Br J Pharmacol 140: 831-838.

Shaw-Lutchman TZ, Barrot M, Wallace T, Gilden L, Zachariou V, Impey $S$ et al (2002). Regional and cellular mapping of cAMP response element-mediated transcription during naltrexoneprecipitated morphine withdrawal. J Neurosci 22: 3663-3672.

Suzuki T, Funada M, Narita M, Misawa M, Nagase H (1993). Morphine-induced place preference in the CXBK mouse: characteristics of mu opioid receptor subtypes. Brain Res 602: $45-52$.

Tsuda K, Tsuda S, Nishio I, Masuyama Y, Goldstein M (1998). Effects of galanin on dopamine release in the central nervous system of normotensive and spontaneously hypertensive rats. Am J Hypertens 11: 1475-1479.

Valjent E, Caboche J, Vanhoutte P (2001). Mitogen-activated protein kinase/extracellular signal-regulated kinase induced gene regulation in brain: a molecular substrate for learning and memory? Mol Neurobiol 23: 83-99.

Valjent E, Corbille AG, Bertran-Gonzalez J, Herve D, Girault JA (2006a). Inhibition of ERK pathway or protein synthesis during reexposure to drugs of abuse erases previously learned place preference. Proc Natl Acad Sci USA 103: 2932-2937. 
Valjent E, Corvol JC, Trzaskos JM, Girault JA, Herve D (2006b). Role of the ERK pathway in psychostimulant-induced locomotor sensitization. BMC Neurosci 7: 20.

Valjent E, Pages C, Herve D, Girault JA, Caboche J (2004). Addictive and non-addictive drugs induce distinct and specific patterns of ERK activation in mouse brain. Eur J Neurosci 19: 1826-1836.

Valjent E, Pascoli V, Svenningsson P, Paul S, Enslen H, Corvol JC et al (2005). Regulation of a protein phosphatase cascade allows convergent dopamine and glutamate signals to activate ERK in the striatum. Proc Natl Acad Sci USA 102: 491-496.

Wang HY, Wild KD, Shank RP, Lee DH (1999). Galanin inhibits acetylcholine release from rat cerebral cortex via a pertussis toxin-sensitive G(i) protein. Neuropeptides 33: 197-205.

Waters SM, Krause JE (2000). Distribution of galanin-1, -2 and -3 receptor messenger RNAs in central and peripheral rat tissues. Neuroscience 95: 265-271.

Widnell KL, Self DW, Lane SB, Russell DS, Vaidya VA, Miserendino MJ et al (1996). Regulation of CREB expression: in vivo evidence for a functional role in morphine action in the nucleus accumbens. J Pharmacol Exp Ther 276: 306-315.

Williams JA, Su HS, Bernards A, Field J, Sehgal A (2001). A circadian output in Drosophila mediated by neurofibromatosis-1 and Ras/MAPK. Science 293: 2251-2256.
Wise RA (1987). The role of reward pathways in the development of drug dependence. Pharmacol Ther 35: 227-263.

Wu WP, Hao JX, Lundström L, Wiesenfeld-Hallin Z, Langel Ü, Bartfai T et al (2003). Systemic galnon, a low-molecular weight galanin receptor agonist, reduces heat hyperalgesia in rats with nerve injury. Eur J Pharmacol 482: 133-137.

Wynick D, Small CJ, Bloom SR, Pachnis V (1998). Targeted disruption of the murine galanin gene. Ann N Y Acad Sci 863: 22-47.

Zachariou V, Brunzell DH, Hawes J, Stedman DR, Bartfai T, Steiner RA et al (2003). The neuropeptide galanin modulates behavioral and neurochemical signs of opiate withdrawal. Proc Natl Acad Sci USA 100: 9028-9033.

Zachariou V, Parikh K, Picciotto MR (1999). Centrally administered galanin blocks morphine place preference in the mouse. Brain Res 831: 33-42.

Zarrindast MR, Bananej M, Khalilzadeh A, Fazli-Tabaei S, HaeriRohani A, Rezayof A (2006a). Influence of intracerebroventricular administration of dopaminergic drugs on morphine statedependent memory in the step-down passive avoidance test. Neurobiol Learn Mem 86: 286-292.

Zarrindast MR, Farahmandfar M, Rostami P, Rezayof A (2006b). The influence of central administration of dopaminergic and cholinergic agents on morphine-induced amnesia in morphinesensitized mice. J Psychopharmacol 20: 59-66. 